\title{
Infliximab-Induced Tubulointerstitial Nephritis with Image Findings of Striated Nephrogram in Crohn's Disease
}

\author{
Tomonori Sato, ${ }^{1}$ Yoshihide Kawasaki, ${ }^{1}$ Akihiro Ito, ${ }^{1}$ Hideaki Izumi, ${ }^{1}$ \\ Naoki Kawamorita, ${ }^{1}$ Shinichi Yamashita, ${ }^{1}$ Koji Mitsuzuka, ${ }^{1}$ Tomonori Matsuura, ${ }^{2}$ \\ Mika Watanabe ${ }^{3}$ and Yoichi Arai ${ }^{1}$ \\ ${ }^{1}$ Department of Urology, Tohoku University Graduate School of Medicine, Sendai, Miyagi, Japan
${ }^{2}$ Department of Diagnostic Radiology, Tohoku University Graduate School of Medicine, Sendai, Miyagi, Japan
${ }^{3}$ Division of Pathology, Tohoku University Hospital, Sendai, Miyagi, Japan
}

Tubulointerstitial nephritis is primary injury to renal tubules and interstititum which could be resulting in decreased renal function. The acute and chronic forms are most often due to allergic drug reactions or to infections. Tubulointerstitial nephritis in Crohn's disease has rarely been reported. Imaging findings of a striated nephrogram on enhanced computed tomography (CT) could represent the clinical state of tubulointerstitial nephritis. This is the first report of tubulointerstitial nephritis caused by infliximab, monoclonal antibody against human tumor necrosis factor- $\alpha$, showing striated nephrograms in Crohn's disease. The case of a 28-year-old man treated with infliximab for Crohn's disease is described. Infliximab was added to his maintenance therapy, and bowel symptoms were stable. The patient presented with a 2-month history of fever and an elevated C-reactive protein after infliximab administration for 4.5 years. Contrast-enhanced CT showed striated nephrograms in both kidneys. Urinalysis showed no abnormal findings. The pathological diagnosis on CT-guided percutaneous renal needle biopsy was drug-induced tubulointerstitial nephritis because of eosinophilic infiltration with neutrophils mainly in the tubulointerstitial areas. The imaging findings of striated nephrogram are important for the diagnosis of tubulointerstitial nephritis. Tubulointerstitial nephritis could be caused by drug-induced inflammation or direct extension of Crohn's disease as an extra-interstitial manifestation. The treatment strategies for these two diseases are contradictory to each other and inappropriate treatment could worsen the renal function. Needle biopsy is therefore indispensable for differential diagnosis.

Keywords: Crohn's disease; infliximab; renal biopsy; striated nephrogram; tubulointerstitial nephritis Tohoku J. Exp. Med., 2018 July, 245 (3), 149-152. (C) 2018 Tohoku University Medical Press

\section{Introduction}

Tubulointerstitial nephritis (TIN) is defined pathologically by inflammatory lesions involving the renal tubules and interstitium. In Crohn's disease, TIN could be caused by drug-induced inflammation or direct extension of Crohn's disease as an extraintestinal manifestation in the kidney (Heidt et al. 2010). The pathological findings of the former are eosinophilic infiltration with neutrophils mainly in the tubules and interstitium. The latter presents with local lymphocyte inflammation and limited infiltration of eosinophils in the interstitium. These differing pathological findings become a key point for deciding the treatment strategy. There have been only two case reports on TIN induced by infliximab in Crohn's disease (Yoo et al. 2014; Ota et al. 2016). The characteristic imaging findings of a striated nephrogram could represent the clinical state of drug-induced TIN. Therefore, urologists should understand the imaging findings of striated nephrogram and the clinical state of TIN.

\section{Case Report}

A 28-year-old man had been treated at our hospital following the diagnosis of Crohn's disease in 2010. Periodic administration of infliximab was initiated from 2011. During maintenance therapy with agents including azathioprine and mesalazine, the intestinal symptoms of Crohn's disease were stable. The patient presented with a 2-month history of a high temperature $\left(\sim 38^{\circ} \mathrm{C}\right)$ and elevated C-reactive protein.

Blood and serum biochemical findings were: white blood cell (WBC) count 9,400/ $\mu \mathrm{L}$; CRP $3.6 \mathrm{mg} / \mathrm{dL}$; blood urea nitrogen (BUN) $10 \mathrm{mg} / \mathrm{dL}$; and creatinine $1.05 \mathrm{mg} / \mathrm{dL}$. A mild inflammatory reaction and renal dysfunction were

Received March 27, 2018; revised and accepted June 20, 2018. Published online July 5, 2018; doi: 10.1620/tjem.245.149.

Correspondence: Yoshihide Kawasaki, M.D., Department of Urology, Tohoku University Graduate School of Medicine, Seiryo-machi, Aoba-ku, Sendai, Miyagi 980-8574, Japan.

e-mail: kawasaki@uro.med.tohoku.ac.jp 
observed. The patient did not present with any signs of allergic reaction, such as skin rashes, liver dysfunction, or eosinophilia in the peripheral blood picture. Urinalysis showed no abnormal findings, such as hematuria, pyuria, or proteinuria. Abdominal contrast-enhanced computed tomography (CT) showed that multiple poor contrast and high absorption regions were oriented parallel to the axis of the tubules and collecting ducts in the excretory phase (Fig. $1)$.

Because the imaging findings were unchanged one month later, and the diagnosis was inconclusive, contrastenhanced CT-guided percutaneous renal needle biopsies of the poor contrast lesions as the main targets were performed.

Pathological findings indicated severe infiltration of inflammatory cells, including neutrophils, lymphocytes, plasma cells, and eosinophils, mainly in the interstitium and renal tubules (Fig. 2). Considering that eosinophils were found accompanied by inflammatory cells, the patient was pathologically diagnosed as having drug-induced TIN. The clinical symptoms were ameliorated 2.5 months after dis- continuation of infliximab. During that period, no relapse was noted while mesalazine administration was continued, leading to the diagnosis of infliximab-induced TIN. The patient is currently under observation, and the renal disorder resolved with a serum creatinine level of $1 \mathrm{mg} / \mathrm{dL}$. Contrast-enhanced CT showed several mild atrophic renal parenchyma lesions 9 months after the diagnosis.

\section{Discussion}

The present patient showed the characteristic findings of a striated nephrogram on enhanced CT. Striated nephrogram is defined as the alternating distribution of poor contrast and high absorption regions oriented parallel to the axis of the tubules and collecting ducts. In Crohn's disease, there is only one report of mesalazine-induced TIN, which showed similar CT findings (Lomboy and Jose 2017). The region with poor contrast is formed by underperfusion resulting from underlying vascular spasm due to interstitial edema. The contrast-enhanced high absorption region is formed by the accumulation and concentration of the contrast agent due to mechanical compression against the col-
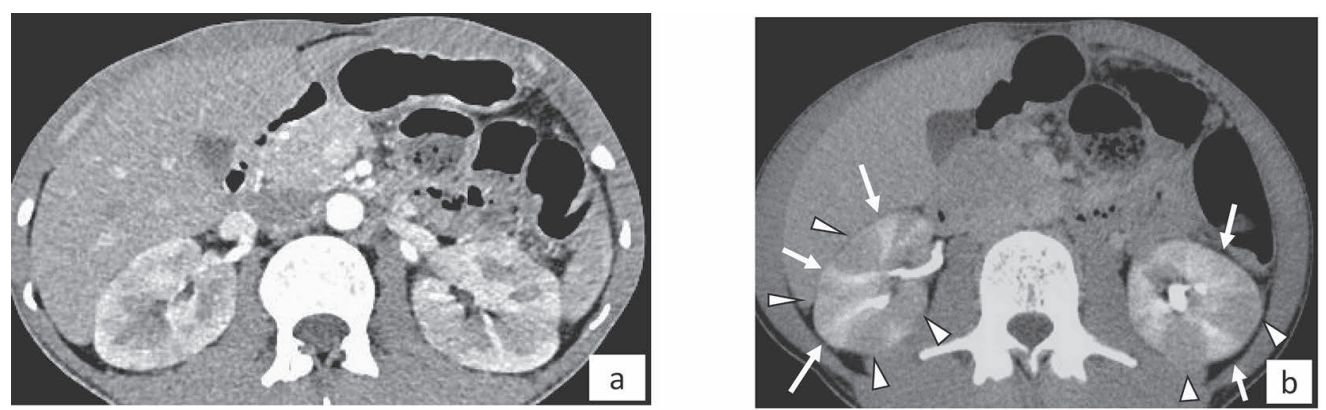

Fig. 1. Contrast-enhanced CT scans.

(a) Early phase showed delayed contrast enhanced renal cortex.

(b) Excretory phase showed striated nephrograms. Poorly contrast enhanced renal paranchyma (white arrowheads) and high absorption regions (white arrows) were observed parallel to the axis of the tubules and collecting ducts.
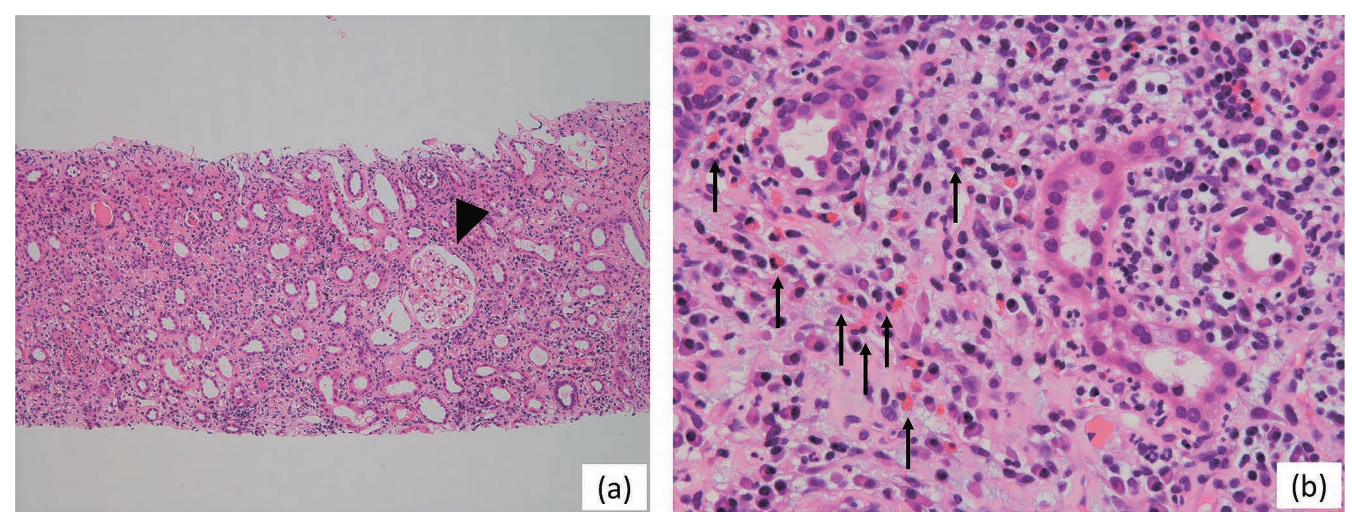

Fig. 2. Pathological findings.

(a) Light microscopy of the renal biopsy specimen shows infiltration of mononuclear cells and neutrophils between tubular epithelial cells and interstitium (Hematoxylin-Eosin staining). No glomerular or vascular changes were seen (an arrowhead).

(b) At high magnification, infiltration of various inflammatory cells, including lymphocytes, plasma cells, eosinophils, and neutrophils was observed (Hematoxylin-Eosin Staining). A higher proportion of eosinophil infiltration (arrows) was noted. 
lecting duct from the tubule associated with interstitial edema, along with drug-induced direct dysfunction of the renal tubule. Regarding patients who present with bilateral striated nephrogram, TIN, acute pyelonephritis, and autosomal recessive polycystic kidney disease have been reported (Moinuddin et al. 2015). The cause of TIN has been noted to be drug-induced, infectious, and idiopathic in $71 \%$, $15.6 \%$, and $7.8 \%$ of cases, respectively (Baker and Pusey 2004).

In Crohn's disease, infliximab was considered to be the causative drug of TIN in two previously reported cases (Yoo et al. 2014; Ota et al. 2016). Yoo et al. (2014) reported that the timing of TIN onset after infliximab administration was 2 years, whereas Ota et al. (2016) reported it was 9 years. Renal biopsies were performed due to renal impairment and TIN was diagnosed by pathological findings in these cases. The present patient also experienced renal dysfunction after infliximab administration for more than 4.5 years. Although infliximab-induced TIN may develop after long-term administration, the reason remains unclear (Ota et al. 2016). Furthermore, the symptoms of the present patient did not resolve until 3 months after discontinuation of infliximab. The two previous reports also indicated that recovery from symptoms took 2-3 months. Based on the half-life of infliximab, 2-3 months after discontinuation of the drug is likely needed to ameliorate symptoms. Ota et al. (2016) reported urinary findings of microscopic hematuria, pyuria and proteinuria. In contrast, neither the presently reported case nor the case reported by Yoo et al. (2014) had these urinary abnormalities. Although the characteristic image findings in our case played an important role in the diagnosis of TIN, the previous reports did not mention image findings.

It is important to distinguish drug-induced TIN from extraintestinal lesions due to Crohn's disease that occur in patients suffering from fever and renal disorder (Marcus et al. 2008; Waters et al. 2008). Extraintestinal manifestation is defined as organ inflammatory processes outside the intestinal tract. Extraintestinal manifestation is found in $6-46 \%$ of all cases of inflammatory bowel disease (Mendoza et al. 2005). Although extraintestinal lesions comprising arthritis, dermatitis, and iritis have mainly been reported, renal lesions are rare. There are several reports of extraintestinal manifestation in the kidney in Crohn's disease. Tubulointerstitial nephritis has been reported in 9 patients, but occurred during follow-up of the bowel disease, and was not associated with drug treatment (Larchet et al. 1988; Archimandritis and Weetch 1993; Tovbin et al. 2000; Izzedine et al. 2002; Marcus et al. 2008; Waters et al. 2008; Shahrani Muhammad et al. 2010). The main mechanisms of extraintestinal manifestation in the kidney are thought to include infectious agents, T-cell-mediated hypersensitivity, circulating bacterial endotoxins, and genetic factors (Colvin et al. 2014).

Contrast-enhanced CT findings of extraintestinal manifestation in the kidney are visualized as localized contrast- deficient areas, and it is difficult to distinguish them from drug-induced TIN based on imaging findings alone. Therefore, pathological findings on renal biopsy examination are very important for making the diagnosis.

The pathology of drug-induced TIN is characterized by diffuse appearance of eosinophils accompanied by inflammatory cells in the renal tubules and interstitium. On the other hand, extraintestinal manifestation in the kidney of Crohn's disease mainly shows lymphocyte infiltration, and eosinophils are not generally seen. These different pathological findings are important to distinguish these two disorders. In addition, there were several reports about glomerulonephritis associated with Crohn's disease. According to a retrospective review of 83 kidney biopsies from patients with inflammatory bowel diseases, the frequency of immunoglobulin (Ig)A nephropathy was significantly higher in those patients than in control cases with renal biopsy specimens during the same period (24\% vs. $8 \%$ ) (Ambruzs et al. 2014). Hence, it is also necessary to exclude the secondary glomerular diseases and vascular lesions originating from Crohn's disease by renal biopsy.

For treatment strategy, suspected drugs should be discontinued in the case of drug-induced TIN. Conversely, the dosage of treatment drugs should be increased in the case with extraintestinal manifestation of Crohn's disease. As the treatment methods for the two cases are conflict, it is important to clarify by renal biopsy whether the systemic symptoms are due to drug-induced TIN or extraintestinal manifestation of Crohn's disease. Precise and early diagnosis could contribute to avoiding renal dysfunction.

In conclusion, the clinical state of drug-induced TIN in Crohn's disease was characterized by a striated nephrogram on contrast-enhanced CT. However, imaging findings of a striated nephrogram require differential diagnosis between TIN and extraintestinal manifestation in the kidney of Crohn's disease. Renal biopsy is essential to clarify these two diseases with different treatments, because inappropriate treatment worsens renal prognosis. Urologists need to understand the imaging findings of a striated nephrogram and the clinical state of TIN.

\section{Conflict of Interest}

The authors declare no conflict of interest.

\section{References}

Ambruzs, J.M., Walker, P.D. \& Larsen, C.P. (2014) The histopathologic spectrum of kidney biopsies in patients with inflammatory bowel disease. Clin. J. Am. Soc. Nephrol., 9, 265-270.

Archimandritis, A.J. \& Weetch, M.S. (1993) Kidney granuloma in Crohn's disease. BMJ, 307, 540-541.

Baker, R.J. \& Pusey, C.D. (2004) The changing profile of acute tubulointerstitial nephritis. Nephrol. Dial. Transplant., 19, 8-11.

Colvin, R.B., Traum, A.Z., Taheri, D., Jafari, M. \& Dolatkhah, S. (2014) Granulomatous interstitial nephritis as a manifestation of Crohn disease. Arch. Pathol. Lab. Med., 138, 125-127.

Heidt, J., Ooms, E.C., van der Werf, S.D. \& Groeneveld, J.H. (2010) Tubulo-interstitial nephritis in inflammatory bowel 
disease: extra-intestinal manifestation or drug toxicity? Ned. Tijdschr. Geneesk., 154, A1647.

Izzedine, H., Simon, J., Piette, A.M., Lucsko, M., Baumelou, A., Charitanski, D., Kernaonet, E., Baglin, A.C., Deray, G. \& Beaufils, H. (2002) Primary chronic interstitial nephritis in Crohn's disease. Gastroenterology, 123, 1436-1440.

Larchet, M., Guillot, M., Mandard, J.C., Boutard, P., Alibert, L., Delmas, P. \& Duhamel, J.F. (1988) Crohn's enteritis and chronic tubulo-interstitial nephropathy in an adolescent. Arch. Fra. Pediatr., 45, 649-651.

Lomboy, J.R. \& Jose, F. (2017) Allergic interstitial nephritis masquerading as pyelonephritis in a pediatric patient with crohn disease. J. Pediatr. Gastroenterol. Nutr., 65, e18-e20.

Marcus, S.B., Brown, J.B., Melin-Aldana, H. \& Strople, J.A. (2008) Tubulointerstitial nephritis: an extraintestinal manifestation of Crohn disease in children. J. Pediatr. Gastroenterol. Nutr., 46, 338-341.

Mendoza, J.L., Lana, R., Taxonera, C., Alba, C., Izquierdo, S. \& Diaz-Rubio, M. (2005) Extraintestinal manifestations in inflammatory bowel disease: differences between Crohn's disease and ulcerative colitis. Med. Clin. (Barc), 125, 297-300.

Moinuddin, I., Bracamonte, E., Thajudeen, B., Sussman, A., Madhrira, M. \& Costello, J. (2015) Allergic interstitial nephritis manifesting as a striated nephrogram. Case Rep. Med., 2015, 250530.

Ota, M., Iwai, H., Imai, K., Kamiya, M., Yoshihashi-Nakazato, Y., Miyasaka, N. \& Kohsaka, H. (2016) Acute tubulointerstitial nephritis associated with infliximab in a patient with Crohn's disease. Intern. Med., 55, 1367-1370.

Shahrani Muhammad, H.S., Peters, C., Casserly, L.F., Dorman, A.M. \& Watts, M. (2010) Relapsing tubulointerstitial nephritis in an adolescent with inflammatory bowel disease without aminosalicylate exposure. Clin. Nephrol., 73, 250-252.

Tovbin, D., Kachko, L. \& Hilzenrat, N. (2000) Severe interstitial nephritis in a patient with renal amyloidosis and exacerbation of Crohn's disease. Clin. Nephrol., 53, 147-151.

Waters, A.M., Zachos, M., Herzenberg, A.M., Harvey, E. \& Rosenblum, N.D. (2008) Tubulointerstitial nephritis as an extraintestinal manifestation of Crohn's disease. Nat. Clin. Pract. Nephrol., 4, 693-697.

Yoo, Y.J., Chung, S.Y., Gu, D.H., Ko, G.J., Pyo, H.J., Kwon, Y.J., Bak, Y.T. \& Won, N.H. (2014) A case of late onset-acute tubulointerstitial nephritis with infliximab and mesalazine treatment in a patient with Crohn's disease. Korean J. Gastroenterol., 63, 308-312. 